\title{
Correspondence
}

\section{Antibodies to HTLV-I in sera from patients with connective tissue diseases}

SIR, It has been suggested that HTLV-I infection may be associated with the development of some connective tissue diseases (CTD), including poly- and dermatomyositis (PM and DM), systemic lupus erythematosus (SLE), scleroderma, and Sjögrens syndrome (SS). ${ }^{12}$ This was based on eight case reports in which neither the ethnic origin of their patients nor the assays used to determine HTLV-I seropositivity were defined. Six cases of PM in HTLV-I antibody positive Jamaicans have also been reported (Rodgers-Johnson et al, quoted in ref 3). These observations prompted us to examine the HTLV-I antibody status of a large cohort of patients with CTD attending a rheumatology clinic in London. A total of 98 serum samples from 80 patients: 42 with SLE, of whom eight also had SS, and 38 with PM/DM, were screened using an HTLV-I enzyme linked immunosorbent assay (ELISA) kit (Dupont Ltd). Most (55) were Caucasian, 16 were of Indian, Middle, or Far East descent, and nine were West Indians. Positive samples were retested using the same assay system, and repeatedly positive sera were further tested by particle agglutination and immunofluorescence on HTLV-I infected cells. A total of $\mathbf{9 8}$ serum samples was tested and the results were as follows: screen positive: 5 , repeat positive: 3 , confirmed positive: 1 . The two serum samples that were positive in the ELISA test on one occasion only were from the same patient, a child with DM; eight other serum samples from this child were negative in the ELISA. The two samples that were consistently positive in the ELISA, but not confirmed positive, were from one patient with PM and one with SLE, both of whom were Caucasian. The only confirmed HTLV-I antibody positive serum was from a West Indian woman with SLE.

Our results confirm the assertion in a recent Lancet editorial ${ }^{4}$ that results of positive HTLV-I antibody tests must be interpreted with caution and it is not acceptable to rely on one assay system alone. This is likely to be of special importance in patients with CTD, in whom the occurrence of antibodies of unusual specificity is common. We found no evidence to suggest that HTLV-I was associated with CTD in our group of patients.

Department of Virology,

W IRVING

John Radcliffe Hospital

Oxford

Virus Reference Laboratory,

P WHITE

Central Public Health Laboratories,

Colindale

Dept of Rheumatology Research, University College and

Middlesex Hospital Medical School, London

\section{References}

1 Vernant J-C, Buisson G G, Sobesky G, Arfi S, Gervaise G, Roman G C. Can HTLV-I lead to immunological disease? Lancet 1987; ii: 404.

2 Vernant J-C, Buisson G, Magdeleine J, et al. T-lymphocyte alveolitis, tropical spastic paresis and Sjogren Syndrome. Lancet 1988; i: 177.

3 Marx J L. Leukemia virus linked to nerve disease. Science 1987; 236: $1059-61$.

4 Anonymous. HTLV-I comes of age [Editorial]. Lancet 1988; i: 217-9.

\section{Anticardiolipin antibodies in systemic lupus erythematosus}

SIR, We read with interest the paper by Gharavi et al on the IgG subclass distribution of anticardiolipin antibodies in systemic lupus erythematosus (SLE). ${ }^{1}$ We have performed a similar study and draw similar conclusions about the presence of anticardiolipin activity in all four sulf classes. We have, however, examined serum samples fro a wider variety of patients than Gharavi and coworkers and. have found significant differences in anticardiolipin sub्ञ class distribution in patients with and without SLE.

Serum samples were obtained from 28 patients with high levels of IgG cardiolipin; 17 had a diagnosis of SLE $\frac{0}{\varnothing}$ or SLE-like illness and the remaining 11 had a variety of $\varnothing$ acute and chronic inflammatory illnesses (three rheuma- $\overrightarrow{\overrightarrow{0}}$ toid arthritis, one chronic active hepatitis, one sarcoid, one $\frac{3}{3}$ lymphoma, one vasculitis, one emphysema, and three probable acute infections). Fourteen of the 17 patients had strong positive IgG antibodies to double stranded DNA (dsDNA) and the remaining three had had dsDNA antibodies at an earlier stage in their illness. (IgG antibodies to dsDNA were measured using an isotype specific enzyme 3 linked immunosorbent assay (ELISA) similar to that described by Gharavi et $a l$ and used routinely in our laboratory.) Results were expressed in arbitrary units. Normals have a mean of $4 \mathrm{U} / \mathrm{ml}$, whereas all our patients showed 음 $>20 \mathrm{U} / \mathrm{ml}$. Sixteen of the 17 patients with SLE were $>$ female. In the non-SLE group no patient had double stranded DNA antibodies and only 1/11 was female.

Isotype and IgG subclass specific anticardiolipin assays $\sigma$ were performed by an ELISA, using a method similar to N that used by Gharavi et al with the following differences: (a) $10 \%$ newborn calf serum in phosphate buffered saline $\omega$ was used as the blocking agent and diluent; $(b)$ patients' sera were diluted 1:10 for the isotype specific assay ando $1: 20$ for the subclass assay; $(c)$ anti-subclass monoclonal $\mathbb{\Phi}$ antibodies used were IgG1 (NL16), IgG2 (SH21), IgG3 $\stackrel{\oplus}{?}$ (BAM08); $(d)$ peroxidase conjugated rabbit antihuman 0 IgG and antimouse IgG (Dako) were used for the final stage in the ELISA; $(e)$ differences in the affinity of the $\mathbb{D}$ monoclonal antibodies for the appropriate subclasses were $\frac{?}{\mathbb{Q}}$ 
assessed by performing ELISAs on plates coated with serial dilutions of IgG1, 2, 3, and 4 myelomas. IgG subclass anticardiolipin levels were expressed as optical density corrected by a factor derived from these standard curves. Ten normal controls were assayed, and optical density values greater than three standard deviations above the control mean were considered positive; $(f)$ each isotype specific ELISA plate incorporated serial dilutions of a high titre standard serum. Patients' sera were compared with the standard curve thus obtained and the results expressed in arbitrary units $(<10 \mathrm{U} / \mathrm{ml}$ is equivalent to three standard deviations above the normal mean. All patients in this series had IgG titres $>25 \mathrm{U} / \mathrm{ml}$ ).

Patients with SLE had significantly higher levels of IgG anticardiolipin than the non-SLE group (SLE mean 98.8, range 26-230; non-SLE mean 42 , range 27-70; $p<0.01$ by Student's $t$ test). Fig. 1 shows the IgG subclass anticardiolipin distributions. There were significantly more serum samples with positive IgG1 and IgG2 titres in the SLE groups $\left(\chi^{2}=5.2, p<0.05\right.$ and $\chi^{2}=10.07, p<0.01$ respectively), but more positive IgG3 titres were found in the non-SLE group $\left(\chi^{2}=12 \cdot 3, p<0 \cdot 001\right)$. Anticardiolipin tended to be mainly IgG1 and IgG2 in the SLE group (either being the major subclass) and IgG1 and IgG3 in the non-SLE group (Table 1).

Table 1 Ratio of $\operatorname{IgG}$ subclasses of anticardiolipin antibodies

\begin{tabular}{lcl}
\hline Ratio & $S L E$ & Non-SLE \\
\hline Anticardiolipin IgG1:IgG2* & 11 & 8 \\
$>1$ & 6 & 2 \\
$<1$ & & \\
Anticardiolipin IgG2:IgG3† & 15 & 2 \\
$>1$ & 0 & 8 \\
$<1$ &
\end{tabular}

Subclass levels were measured as optical density corrected by a factor derived from standard curves produced with purified IgG1-4 myelomas.

$\chi^{2}=0.7, p>0.05$.

$+\chi^{2}=17 \cdot 6, p<0 \cdot 001$. $\triangle:$ SLE $n=11$

$\triangle:$ NON SLE $n=11$

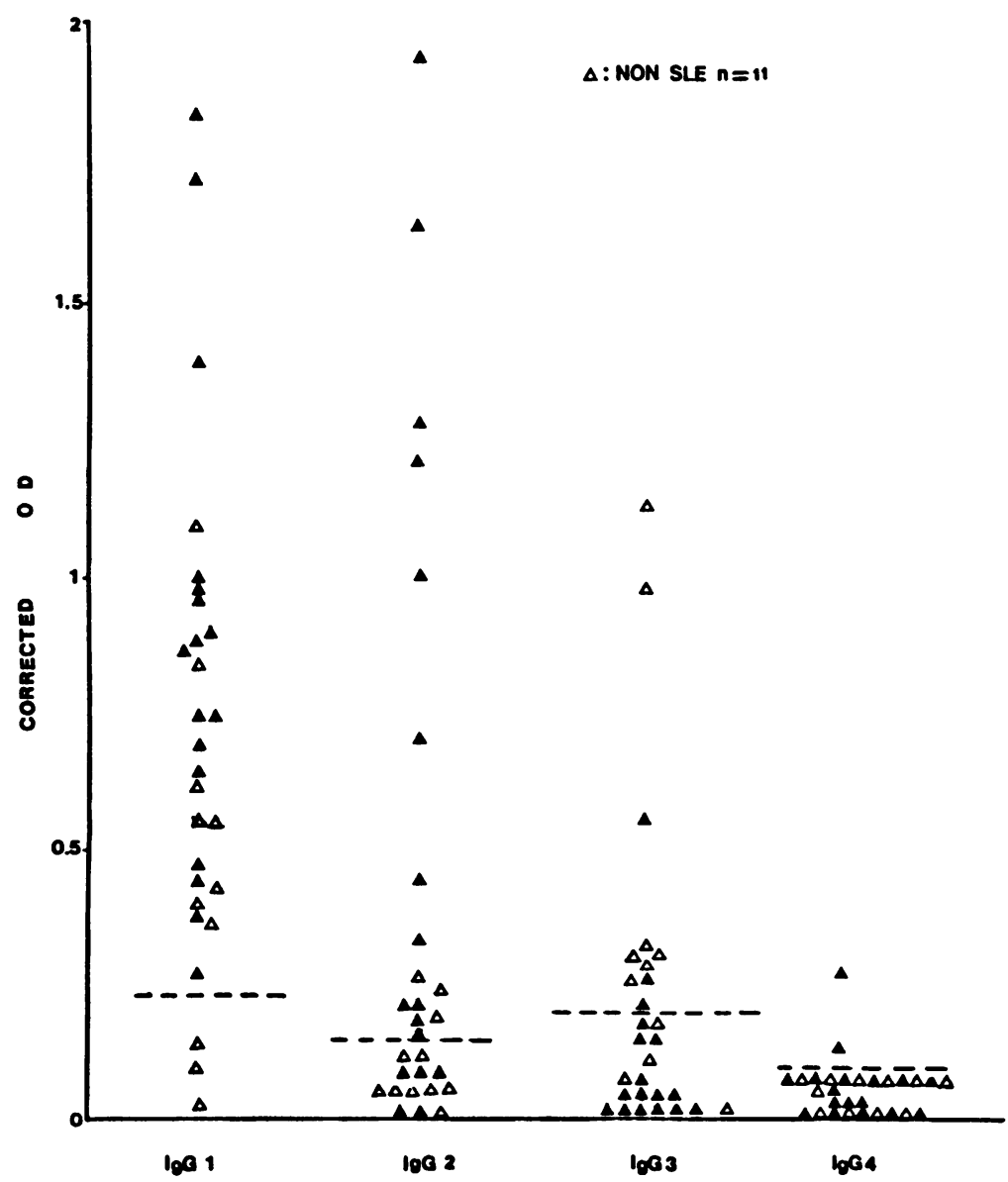

Fig. 1 IgG subclass of anticardiolipin antibodies in 17 patients with systemic lupus erythematosus and 11 with other disorders. Horizontal dashed lines indicate three standard deviations above the mean obtained from 10 normals. 
These results support and extend the observations of Gharavi et al. ${ }^{1}$ They provide further evidence that antidsDNA and anticardiolipin antibodies are to some extent discrete populations in the patients with principally IgG1 and IgG3 anticardiolipin binding - that is, the principal subclasses of IgG anti-dsDNA, showed no DNA binding.

These results also emphasise the heterogeneity of anticardiolipin/antiphospholipid antibodies: patients with and without SLE show different affinities for different phospholipids, ${ }^{2}$ different effects on in vitro coagulation studies, ${ }^{3}$ and have different clinical correlations. ${ }^{3}$ It would also seem that they show different IgG subclass distribution. We feel it is unlikely that the difference between our groups reflects either the marked sex difference or the higher total IgG anticardiolipin in the SLE groups: the subclass ratios within each group were similar for both high and low total IgG anticardiolipin and for both men and women. Data from our laboratory suggest that the IgG subclass distribution of these sera is the same for the binding of three other phospholipids with widely differing charge: phosphatidylserine, phosphatidylinositol, and phosphatidylethanolamine (Snowden et al, unpublished data).

Regional Immunology Service, St Mary's Hospital,

Hathersage Road,

NEIL SNOWDEN

Manchester M3 0JH

\section{References}

1 Gharavi A E, Harris E N, Lockshin M D, Hughes G R V, Elkon K B. IgG subclass and light chain distribution of anticardiolipin and anti-DNA antibodies in systemic lupus erythematosus. Ann Rheum Dis 1988; 47: 286-90.

2 Colaco C B, Male D K. Anti-phospholipid antibodies in syphilis and a thrombotic subset of SLE distinct profiles of epitope specificity. Clin Exp Immunol 1985; 59: 449-56.

3 Harris E N, Gharavi A E, Hughes G R V. Anti-phospholipid antibodies. Clin Rheum Dis 1985; 11: 591-609.

\section{Lyme arthritis in Spain}

SIR, We have been interested in the increasing recognition of Lyme arthritis in Europe reported by Huaux et al. ${ }^{1}$ The present case is the first described in Spain and has the peculiarity that synovial swelling recovered rapidly with ceftriaxone sodium after an unsuccessful 10 day penicillin regimen.

In October 1986 a 62 year old man had a cutaneous eruption, which retrospectively was compatible with erythema chronicum migrans. In January 1987 he was admitted to the hospital because of a two month history of mild fever, right lower abdominal pain, and generalised polyarticular pain followed by right knee synovial swelling and weight loss. His epidemiological antecedents were unremarkable except for his job as a rag picker.

Physical examination showed fever of $38^{\circ} \mathrm{C}$ and signs of active synovitis in the right knee. Laboratory tests dis- closed a sedimentation rate of $104 \mathrm{~mm}$ after the first hour, 믈 white blood cell count of $12 \times 10^{4} / 1$ with normal differential count, and platelet count of $293 \times 10^{9} / 1$. Titres of rheuma- $\overrightarrow{\vec{*}}$ toid factor and antinuclear antibodies as well as cryoglo- bulins and complement levels were normal. Total serum? protein level was $78 \mathrm{~g} / 1, \mathrm{IgG} 2210 \mathrm{~g} / \mathrm{l}, \mathrm{IgM} 610 \mathrm{~g} / \mathrm{l}$, and $\mathrm{IgA}$ 음 220 g/l (normal range: IgG 639-1349 g/l; IgM 56-352 g/l; IgA $70-312 \mathrm{~g} / \mathrm{l})$. The IgM and IgG circulating immune complexes were 80 and $222 \mathrm{mg} / 1$ respectively (normalo range: IgM 10-93 mg/l; IgG 14-147 mg/l). Lumbar? puncture yielded a normal cerebrospinal fluid. Examina- $\overrightarrow{0}$ tion of the synovial fluid showed 3500 leucocytes with $70 \%$ neutrophils. Cultures of the blood, cerebrospinal fluid, andw synovial fluid were negative. Serological tests for Yersinia enterocolitica, Treponema pallidum, Brucella melitensis, $\overline{0}$ Coxiella burnetii, Toxoplasma gondii, Epstein-Barr virus, cytomegalovirus, and hepatitis $B$ virus were negative. The tuberculin skin test (5 TU of PDD-T) was positive $(10 \times 10 \mathrm{~mm})$, but cultural identification of Mycobacterium tuberculosis in Löwenstein-Jensen medium from sputum, cerebrospinal fluid, and synovial biopsy tissue was negative. The following examinations gave normal results:chest roentgenogram, electrocardiogram, barium swallow, gastrointestinal endoscopy, abdominal computed tomo-ว graphy scan, kidney arteriogram, electromyography, and biopsies of the temporal artery, muscle, liver, and synoviak membrane. Serum antibodies against Borrelia burgdorfer $\overrightarrow{\mathrm{k}}$ detected by indirect immunofluorescence were positive $a_{0}^{\circ}$ dilutions of $1 / 32,1 / 128$, and $1 / 256$ at three week intervas. The synovial fluid titre was positive at $1 / 256$. The borr strain was supplied by the Institute Pasteur, Paris. Speciffic IgM antibodies in either serum or synovial fluid were noб tested. The patient was treated with intravenous penicillinמ̆ (20 MU/day for 10 days) and recovered completely excep $\mathbb{D}$ for synovial swelling, which promptly disappeared after administration of intramuscular ceftriaxone sodium? ( $2 \mathrm{~g} /$ day for 14 days). The interval between treatment with penicillin and administration of ceftriaxone sodium was three months.

The clinical spectrum of Lyme disease ranges from asymptomatic to protean manifestations. ${ }^{2}$ Antigeniê̊ variations among borrelia isolates may account for differ옥 ences in the clinical expression of the disease. ${ }^{34}$ Arthritis seems to be less severe and less frequent in Europe than iro the USA. ${ }^{5-7}$ Borrelia's vectors are Ixodes dammini, $\bar{\gtrless}$ pacificus, and I scapularis in America ${ }^{8}$ and I ricinus in Europe, ${ }^{910}$ but other currently unrecognised vectors wilp probably be identified in the future. ${ }^{8}$ In epidemiologicat surveys of Mediterranean spotted fever ${ }^{11} 525$ ticks were collected from our hospital catchment area in the following proportions: Rhipicephalus sanguineus $82.4 \%, R$ turanicus $14.4 \%, R$ pusillus $3 \cdot 2 \%$, and $R$ bursa $3.2 \%$, but no I ricinus were found. We do not know the particular vector in the case here presented. The patient is a resident of the countए Vallés Occidental ( 25 miles west of Barcelona city) with n⿺ history of journeys in Spain or abroad.

Clinical and epidemiological features of Lyme disease io the Mediterranean countries are poorly known because few cases have so far been reported. ${ }^{12}$ The present auto chthonous case illustrates the usefulness of ceftriaxon sodium when response to classical intravenous penicillie treatment is not achieved. ${ }^{13}$ 